\title{
Three-state dual spin valve structure
}

\author{
B. S. Chun*, C. Fowley, M. Abid and J.M.D. Coey \\ School of Physics and CRANN, Trinity College, Dublin, Ireland.
}

\begin{abstract}
A dual spin valve is built with exchange bias on the top and bottom pinned layers and a central free layer. By suitable choice of the antiferromagnetic and ferromagnetic layer thicknesses, it is possible to separate the three magnetization switching fields and produce a staircase magnetoresistive curve. The maximum magnetoresistance ratio is $7.6 \%$ for currentperpendicular-to-plane and $6.3 \%$ for current-in-plane geometries with intermediate magnetoresistances of $3.9 \%$ and $3.0 \%$ respectively. The use of exchange bias in a multistate memory device is discussed.
\end{abstract}

Keywords: Giant magnetoresistance, dual spin valve, exchange bias, multistate memory

PACS Numbers: 75.47.De

* Present address: Department of Materials Science and Engineering, Moscow, Idaho, United States of America.

1. Introduction

Spin valve structures have found applications in high density read heads, programmable logic devices, and nonvolatile magnetic random access memory (MRAM) applications. ${ }^{1-4}$ The basic spin valve (SV) element consists of two ferromagnetic layers separated by a non magnetic spacer 
layer ${ }^{5}$. There are two limiting magnetic configurations in such a device, where the ferromagnetic layers are aligned parallel or antiparallel, which corresponds to a low and high resistance state respectively. One of the ferromagnetic layers is often coupled to an adjacent antiferromagnetic layer which provides exchange bias so that its hysteresis loop is not centered at zero field but is offset by a field of $10-100 \mathrm{mT}$. This is the 'pinned' layer, whereas the other ferromagnetic layer is 'free', as its magnetization will switch around zero field. The spin-polarized current through the conducting spacer layer depends on the relative orientation of the magnetic moments of the electrodes. The giant magnetoresistance (GMR) ratio in a spin valve with a conducting spacer layer is defined as $\left(R_{A P}-R_{P}\right) / R_{P}$, where $R_{P}$ and $R_{A P}$ are the resistances when the magnetizations of the two ferromagnetic layers are aligned parallel and antiparallel, respectively. Accordingly, one bit of binary information can be stored in a SV cell which switches between these two states.

The principal advantage of magnetic random access memory over conventional semiconductor random access memory is nonvolatility. The growing interest of magnetic random access memory has arisen from the large magnetoresistive signals that were predicted ${ }^{6,7}$ and later observed $^{8-10}$ in magnetic tunnel junctions with crystalline $\mathrm{MgO}$ barrier layers. The possibility of spin-torque-transfer (STT) magnetization switching ${ }^{11}$ in these structures ${ }^{12}$ where data is written by passing a spin-polarized current through a thin magnetic layer, which is the free layer in a spin valve structure, has also generated interest in these devices. Unlike half-select MRAM, ${ }^{13}$ where the spin valve structure is sandwiched between current carrying bit and word lines which 
create an Oersted field to switch the free layer element between parallel and antiparallel states, STT switching remains effective as the device dimensions are scaled down. In the Oersted field switching scheme, a larger current density in the bit and word lines is needed to write a bit as the device dimensions are reduced. The amount of information that can be stored in a cell can also be increased, making use of the third spatial dimension. This may be done in a vertical shift register, the magnetic 'racetrack' memory. ${ }^{14}$

Another approach is to increase the number of discrete memory states that can be stored in resistance states of the cell. Binary memory states currently dominate but multistate memory has been receiving much attention, because it offers excellent storage density. ${ }^{15-17}$ Although the possibility of fabricating a multistate magnetic device has been demonstrated, these devices, are however, hard to adapt for practical use because of the inability to selectively switch single elements in a multi-level stack. In a half-select MRAM switching scheme, the field from bit and word lines disturbs more than one element in the stack. Multilevel switching by STT has been demonstrated in an MTJ with a two domain free layer. ${ }^{18}$ Microwave assisted switching has been proposed and allows for selective switching of embedded elements ${ }^{19}$, but it is reliant on the spintorque-oscillator ${ }^{20}$ which is not yet a fully practical proposition.

Here, we take a different approach by using a dual spin valve (DSV) structure with current-inplane (CIP) or current-perpendicular-to-plane (CPP) geometry to create a multistate memory cell. The main point of the DSV structure is that the free layer shows completely independently magnetization switching, and the two pinned layers switch at different applied magnetic fields 
resulting in three resistance states. ${ }^{21,22}$ The multiple states are achieved by controlling the exchange coupling between both ferromagnetic pinned layers and the adjacent antiferromagnetic pinning layers.

\section{Experimental Methods}

The dual spin valve was of the form; $\mathrm{Si} / \mathrm{SiO}_{2} / \mathrm{Buffer} / \mathrm{IrMn} 6 / \mathrm{CoFe} 3.5 / \mathrm{Cu} 2.8 / \mathrm{CoFe} 2.5 / \mathrm{Cu}$ 2.8/CoFe 2.5/IrMn 10/Cap (thickness in $\mathrm{nm}$ ). The buffer layer was Ta 5/CoFe 3/Cu 3. The capping layer was Ta 5. The stack is shown in Fig. 1. Target compositions were $\mathrm{Co}_{90} \mathrm{Fe}_{10}$ and $\mathrm{Ir}_{22} \mathrm{Mn}_{78}$ in atomic percent. Samples were prepared using a Sputtered Films Inc. 'Shamrock' deposition tool with a typical base pressure of less than $3 \times 10^{-9}$ mbar. The free and pinned layer easy axes were aligned along the same direction by applying a $10 \mathrm{mT}$ magnetic field during deposition. Post deposition annealing was carried out $e x$-situ at $250^{\circ} \mathrm{C}$ in a $5 \times 10^{-8}$ mbar vacuum under an applied field of $800 \mathrm{mT}$ for 2 hours. A probe station with an electromagnet capable of generating a magnetic field of $\pm 180 \mathrm{mT}$ was used to measure the magnetoresistive properties of the dual spin valves. CIP measurements were carried out using a standard 4-point probe technique in a linear geometry. Lateral sample size was approx $20 \mathrm{~mm}$ x $20 \mathrm{~mm}$, and inter-probe distance was less than $5 \mathrm{~mm}$. A current of $1 \mathrm{~mA}$, applied parallel to the field, was used to probe the resistance as a function of applied magnetic field. CPP measurements were carried out on nanopillar samples fabricated by electron beam lithography in a FEI Dual Beam system operating at $30 \mathrm{kV}$. Devices of $250 \mathrm{~nm}$ x $400 \mathrm{~nm}$ were fabricated using TOK negative e-beam resist at a dose of $30 \mu \mathrm{C} / \mathrm{cm}^{2}$. Post exposure lift-off was carried out after $\mathrm{Al}_{2} \mathrm{O}_{3}$ deposition to 
insulate the top and bottom contacts. A top contact of Ta 5/Ag 50/Ta 5 was deposited by sputtering and subsequent lift off. The magnetic properties of the dual spin valve structure were characterized by using a Quantum Design Superconducting Quantum Interference Device magnetometer (SQUID).

\section{Results and Discussion}

Before implementing the dual spin valve, we characterized the exchange bias and magnetoresistance (MR) of single spin valve structures with one free and one pinned ferromagnetic layer, with either top or bottom pinning, as a function of layer thickness. To check the antiferromagnetic layer thickness dependence of exchange bias and $\mathrm{MR}$, we made $\mathrm{SiO}_{2} / \mathrm{Ta}$ 5/NiFe 3.5/CoFe 5/Cu 2.8/CoFe 3.5/IrMn $t / \mathrm{Ta} 5(\mathrm{~nm})$ and $\mathrm{SiO}_{2} / \mathrm{Ta} 5 / \mathrm{CoFe} 3 / \mathrm{Cu} 3 / \mathrm{IrMn} t / \mathrm{CoFe}$ 3.5/Cu 2.8/CoFe 5/Ta $5(\mathrm{~nm})$ stacks for the top and bottom pinning, respectively. Also, to check the ferromagnetic layer thickness dependence of exchange bias and MR ratio, we made $\mathrm{SiO}_{2} / \mathrm{Ta}$ 5/NiFe 3.5/CoFe 5/Cu 2.8/CoFe t/IrMn 10/Ta 5 (nm) and $\mathrm{SiO}_{2} / \mathrm{Ta} 5 / \mathrm{CoFe} 3 / \mathrm{Cu} 3 / \mathrm{IrMn} 6 / \mathrm{CoFe}$ t/Cu 2.8/CoFe 5/Ta $5(\mathrm{~nm})$ top and bottom pinned stacks, respectively.

Fig. 2 (a) shows the antiferromagnetic thickness dependence of the exchange bias and MR ratio. Below a $4 \mathrm{~nm}$ thickness of antiferromagnetic $\mathrm{IrMn}$, there is no effective exchange bias. The exchange bias appears at an antiferromagnetic layer thickness of $4 \mathrm{~nm}$ and it continues to increase up to a maximum at a layer thickness of $6 \mathrm{~nm}$ or $10 \mathrm{~nm}$, where it reaches a value of 46 $\mathrm{mT}$ and $31 \mathrm{mT}$ for bottom or top pinning, respectively. Beyond the maxima, the exchange bias decreases with antiferromagnetic layer thickness. The dependence of exchange bias on the 
antiferromagnetic layer thickness is similar to work previously reported. ${ }^{23}$ Exchange bias results from an interfacial interaction between the ferromagnetic and the antiferromagnetic layer. This may be influenced by crystal structure, ${ }^{25}$ magnetic domains,${ }^{26}$ antiferromagnetic grain size ${ }^{23}$, and interface roughness. ${ }^{24}$ The authors of Ref. 23 the authors explain a curve very similar to that in Fig 2 (a) in terms of a model where the exchange bias depends critically on the antiferromagnetic IrMn grain size. The smallest antiferromagnetic particles are not blocked and do not contribute to the exchange bias, the axis of the largest particles cannot be set because the anisotropy energy is too great and it is only IrMn particles with a grain of 8-10 $\mathrm{nm}$ which are effective for exchange bias.

The main reason for larger exchange bias in bottom pinned structures than top pinned structures, is the enhancement of the texture, grain size and morphology of the subsequent layers associated with using the $\mathrm{CoFe}$ and $\mathrm{Cu}$ buffer layer. ${ }^{26}$ The exchange bias field is inversely proportional to the ferromagnetic layer thickness in both cases, as shown in Fig. 2 (b).

Examples of the magnetoresistance curves for top pinned and bottom pinned structures are shown in Figure 3, all measurements are made in the CIP geometry. Fig. 3 (a) and (b) show data for an optimized top pinned device with CoFe 2.5/IrMn $10(\mathrm{~nm})$ and bottom pinned device with an $\mathrm{IrMn} 6 / \mathrm{CoFe} 3.5(\mathrm{~nm})$, respectively.

Results on the dual spin valve structure are shown in Fig. 4, the thickness of the two ferromagnetic $\mathrm{CoFe}$ pinned layers and the antiferromagnetic IrMn pinning layers are chosen to give distinctly different exchange bias fields. The dual spin valve structure clearly exhibits three 
states with different resistance values. Fig. 4 (a) shows the MR curve measured in the CIP geometry, and the magnetization switching of each layer can be clearly distinguished. This is because the free layer switching near zero field to provide a high resistance state, and the two pinned layers switching at different applied reverse fields. In order to give a clear picture of the magnetic moment configurations, the magnetization direction of each magnetic layer is represented by coloured arrows in the figure. The red, black, and blue arrows indicate the magnetization direction of the top pinned, free and bottom pinned CoFe layers, respectively. Starting from the positive saturation field, the magnetization directions of all layers are parallel to the applied field, which results in minimum resistance. As the negative field is increased to near $3 \mathrm{mT}$, the resistance dramatically increases, because the magnetization direction of the central free layer reverses to an antiparallel orientation with respect to that of both pinned layers, resulting in the maximum resistance. As the negative field is increased to near $20 \mathrm{mT}$, the magnetization direction of the top pinned $\mathrm{CoFe}$ layer starts to reverse and is aligned parallel with free layer but antiparallel to the bottom pinned CoFe layer, resulting in an intermediate resistance. Since the bottom pinned CoFe layer has higher exchange bias than that of the top pinned $\mathrm{CoFe}$ layer, which was characterized in Fig. 2, tuning of the exchange coupling strength to give well separated switching fields for both pinned layers is possible. This allows the intermediate resistance state to be stable in field regardless of whether the sweep is started from positive or negative saturation field. By further increasing applied magnetic field beyond $60 \mathrm{mT}$, the magnetization direction of the bottom pinned $\mathrm{CoFe}$ layer rotates towards parallel orientation with 
respect to that of the free and top pinned $\mathrm{CoFe}$ layer and resulting again in the low resistance state.

For clarity the three magnetoresistance states which could potentially give three states at zero field are labeled A, B and C in Fig. 4 (a). A, B, and C can be thought of as corresponding to "0", " 1 " and " 2 " in terms of memory storage. Since the intermediate state at point $\mathrm{C}$ is only stable in an applied field this device cannot function as a three state memory. In order to function as a memory device all three states (“0”, “1”, and "2”) must normally be stable in zero field.

Fig. 4 (b) shows the magnetization curves of the dual spin valve structure as a function of the applied magnetic field along the easy axis. The magnetization switching characteristics agrees well with the resistance results in Fig. 4 (a). There is one extra switch visible in the magnetization curve, compared to Fig. 4 (a), at near zero field. This results from magnetization switching of CoFe buffer layer just above Ta seed layer. The GMR effect results from spin dependent scattering of the conduction electrons in the bulk and/or at the interface of the facing pairs of ferromagnetic layers. The CoFe underlayer does have any influence on the GMR.

Results obtained for the dual spin valve in the CPP geometry in a $250 \mathrm{~nm}$ x $400 \mathrm{~nm}$ nanopillar are shown in Fig. 5. As can be seen from the figure, in the CPP configuration the CPP-GMR ratio increased from $6.3 \%$ to $7.6 \%$ and the resistance value decreased from $2.7 \mathrm{ohm}$ to $0.93 \Omega$ compared to the CIP configuration. The higher GMR ratio in CPP configuration is related to the fact that the spin diffusion length is the important parameter in this geometry, and the electrons are forced to cross all interfaces during transport through the device. In case of CIP case, the 
important parameter is the mean free path of conduction electrons since current is shunted by the normal metal layer, which cannot contribute to GMR. The thicker the nonmagnetic spacer, the lower the GMR. The small resistance value results from the much smaller active volume that is probed using the CPP geometry. Switching by spin-torque-transfer should also be possible in the CPP geometry but our pillar was too large to observe it. The main advantage of the CPP geometry is that the GMR ratio increases with decreasing cell size.

Although multiple resistance states are achieved in our device by optimizing the exchange coupling between the two ferromagnetic pinned layers and the two antiferromagnetic pinning layers, the structure in its present form cannot operate as a multibit memory, because only two of the three states are stable in zero magnetic field. The intermediate resistance state is only accessible in an applied field. Even if the two pinned layers were pinned in opposite directions, there would only be two storage states, corresponding to the two orientations of the free layer. In order to achieve multilevel storage in this type of structure, at least one of the antiferromagnetic pinning layers has to be removed. It has previously been demonstrated, using three free layers with differing coercivities, that a multilevel memory can be constructed without exchange pinning. ${ }^{12}$ If just one of the pinning layers is removed, we have a dual spin valve with two free layers, one half of the device making an exchange biased spin valve, and the other half making a pseudo spin valve with two switchable layers with different coercivity. Provided the magnetoresistance is different for the two halves, the device has four different resistance states in zero field. For example, supposing for the sake of argument that the MR of the exchange biased 
spin valve is $3 \%$ and that the pseudo spin valve is $6 \%$, the device exhibits a staircase of four resistance states according to the previous magnetic field history of the structure. The value of the resistance states being determined by parallel and series resistance laws for CIP and CPP geometries, respectively.

\section{Conclusions}

We have built a dual spin valve structure with distinct switching fields for the two pinned layers, which is a device with a staircase of three distinct magnetoresistive states. The multiple resistance states are achieved by controlling the exchange coupling between both two ferromagnetic pinned layers and adjacent antiferromagnetic pinning layers. As it stands, the structure no application as a multibit since the intermediate resistance state is only available in an applied field. However, if either of the top or bottom pinned layer is unpinned, it would switch about zero with the free layer, and a four-state dual spin valve structure can be achieved.

\section{Acknowledgements}

This work was supported by Science Foundation Ireland as part of the MANSE project. One author (M. Abid) received a Marie Curie Institutional Fellowship at CRANN. We are grateful to M Venkatessan for the SQUID measurements. 


\section{References}

1. Prinz, G.A., Magnetoelectronics, Science 282, 1660-1663 (1998).

2. Wolf, S.A., Awschalom, D.D., Burhman, R.A., Daughton, J.M., von Molnar, S., Chtchelkanova, A.Y., Treger, D.M., Spintronics: A spin-based electronics vision for the future, Science 294, 1488-1495 (2001).

3. Moodera, J.S., LeClair, P., A quantum leap, Nature Materials, 2, 707-708 (2003).

4. Ney, A., Pampuch, C., Koch, R., Ploog, K.H., Programmable computing with a single magnetoresistive element, Nature 425, 485-487 (2003).

5. Dieny, B., Speriosu, V.S., Gurney, B.A., Parkin, S.S.P., Wilhoit, D.R., Roche, K.P., Metin, S., Peterson, D.T., Nadimi, S., Spin Valve Effect in soft ferromagnetic sandwiches, Journal of Magnetism and Magnetic Materials, 93, 101 (1991).

6. Butler, W.H., Zhang, X.G., Schulthess, T.C., MacLaren, J.M., Spin-dependent tunneling conductance of Fe/MgO/Fe sandwiches, Physical Review B, 63, 054416 (2001).

7. Mathon, J., Umerski, A., Theory of tunneling magnetoresistance of an epitaxial Fe/MgO/Fe(001) junction, Physical Review B, 63, 220403 (2001).

8. Parkin, S.S.P., Kaiser, C., Panchula, A. Rice, P.M., Hughes, B., Samant, M., Yang, S.-H., Giant tunnelling magnetoresistance at room temperature with $\mathrm{MgO}$ (100) tunnel barriers, Nature Materials, 3, 862-867 (2004).

9. Ikeda, S., Hayakawa, J., Ashizawa, Y., Lee, Y. M., Miura, K., Hasegawa, H., Tsunoda, M., Matsukura, F., Ohno, H., Tunnel magnetoresistance of $604 \%$ at $300 \mathrm{~K}$ by suppression of Ta 
diffusion in $\mathrm{CoFeB} / \mathrm{MgO} / \mathrm{CoFeB}$ pseudo-spin-valves annealed at high temperature, Applied Physics Letters, 93, 082508 (2008).

10. Slonczewski, J.C., Current-driven excitation of magnetic multilayers, Journal of Magnetism and Magnetic Materials, 159, L1-L7 (1996).

11. Diao, Z., Li, Z., Wang, S., Ding, Y., Panchula, A., Chen, E., Wang, L.-C., Huai, Y., Spintransfer torque switching in magnetic tunnel junctions and spin-transfer torque random access memory, Journal of Physics: Condensed Matter, 19, 165209-165021 (2007).

12. Jeong, W.C., Lee, B.I., Joo, S.K., Three level, six state multilevel magnetoresistive RAM (MRAM), Journal of Applied Physics, 85, $4782-4784$ (1999).

13. Dave, R.W., Steiner, G., Slaughter, J.M., Sun, J.J., Craigo, B., Pietambaram, S., Smith, K., Grynkewich, G., DeHerrera, M., Akerman, J., Tehrani, S., MgO-based tunnel junction material for high-speed toggle magnetic random access memory, IEEE Transactions on Magnetics, 42, 1935-1939 (2006).

14. Parkin, S.S.P., Hayashi, M., Thomas, L., Magnetic domain-wall racetrack memory, Science 320, 190 (2008).

15. Zheng, Y.K., Wu, Y.H., Guo, Z.B., Han, G.C., Li, K.B., Qiu, J.J., Xie, H., Luo, P., Multistate per-cell magnetoresistive random-access memory written at Curie point, IEEE Transactions on Magnetics, 38, 2850-2852 (2002).

16. Lim, C.K., Kim, Y.S, Park, N.Y., Lee, J., Multibit MRAM using a pair of memory cells, IEEE Transactions on Magnetics, 41, 2670-2674 (2005). 
17. Ventura, J., Pereira, A.M., Arujo, J.P., Zhang, Z., Liu, Y., Freitas, P.P., Three-state memory combining resistive and magnetic switching using tunnel junctions, Journal of Physics D: Applied Physics 40, 5819-5823 (2007).

18. Lou. X., Goa, Z., Dimitrov, D.V., Tang, M.X., Demonstration of multilevel cell spin transfer switching in MgO magnetic tunnel junctions, Applied Physics Letters, 93, 242502 (2008).

19. Zhu, J-G., Zhu, X., Tang, Y., Microwave Assisted Magnetic Recording, IEEE Transactions on Magnetics, 44,pg 125 (2008).

20. Devolder, T., Meftah, A., Ito, K., Katine, J.A., Spin transfer oscillators emitting microwave in zero applied magnetic field, Journal of Applied Physics, 101, 063916 (2007).

21. Childress. J.R., Carey, M.J., Cyrille, M.-C., Carey, K., Smith, N., Katine, J.A., Boone, T.D., Driskill-Smith, A.A.G., Maat, S., Mackay, K., Tsang, C.H., Fabrication and recording Study of All-Metal Dual-Spin-Valve CPP Read Heads, IEEE Transactions on Magnetics, 42, 2444 (2006).

22. Law, R., Tan, E.-L., Sbiaa, R., Liew, T., Chong, T.C., Reduction in critical current for spin transfer switching in perpendicular anisotropy spin valves using an in-plane spin polarizer, Applied Physics Letters, 94, 062516 (2009).

23. Vallejo-Fernandez, G., Fernandez-Outon, L.E. and O’Grady, K., Antiferromagnetic grain volume effects in metallic polycrystalline exchange bias systems, Journal of Physics D: Applied Physics, 41, 112001 (2008).

24. Ali. M., Marrows, C.H., Al-Jawad, M., Hickey, B.J., Misra, A., Nowak, U., Usadel, K.D., 
Antiferromagnetic layer thickness dependence of the $\operatorname{IrMn} / \mathrm{Co}$ exchange-bias system, Physical Review B, 68, 214420 (2003).

25. Takano, K., Kodama, R.H., Berkowitz, A.E., Cao, W., Thomas, G., Interfacial Uncompensated Antiferromagnetic Spins: Role in unidirectional anisotropy in polycrystalline $\mathrm{Ni}_{81} \mathrm{Fe}_{19} / \mathrm{CoO}$ bilayers, Physical Review Letters, 79, 1130-1133 (1997).

26. Nogués, J., Lederman, D., Large exchange bias and its connection to interface structure in $\mathrm{FeF}_{2}-\mathrm{Fe}$ bilayers, Applied Physics Letters, 68, 3186-3188 (1996).

27. Yagami, K., Tsunoda, M., Takahashi. M., Enhancement of exchange bias in MnIr/CoFe based spin valves with an ultrathin $\mathrm{Cu}$ underlayer and in situ MnIr surface modification, Journal of Applied Physics, 89, 6609-6611 (2001). 


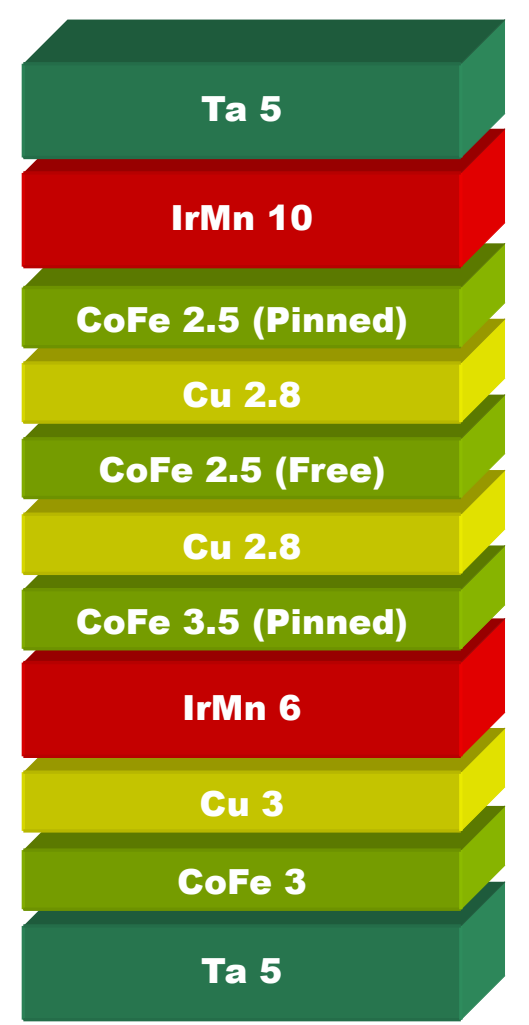

Figure 1 The dual spin valve stack. 


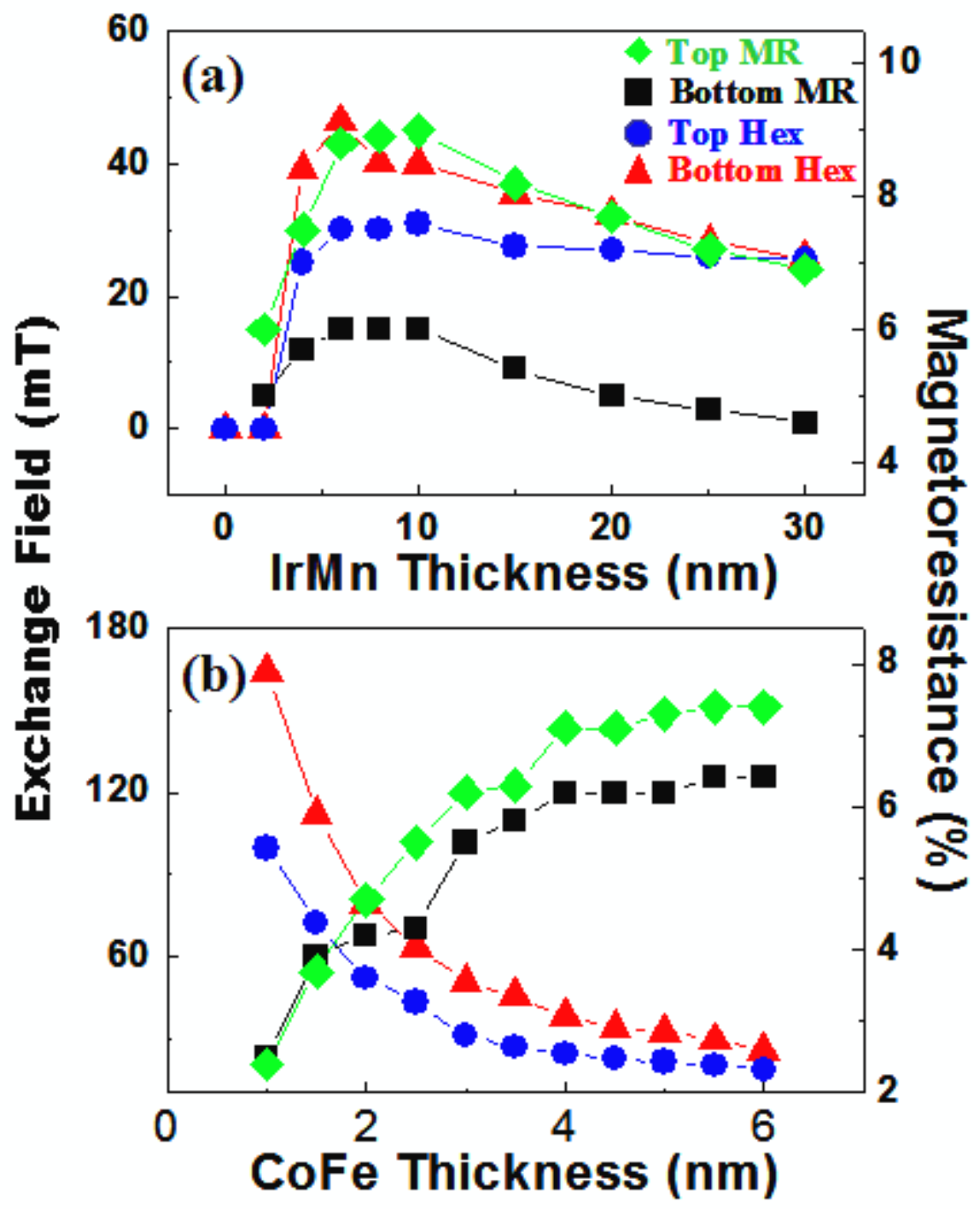

Figure 2. Variation of the exchange bias and magnetoresistive values (a) with antiferromagnetic layer thickness for a fixed ferromagnetic thickness of $3.5 \mathrm{~nm}$ and (b) with ferromagnetic layer thickness for a fixed antiferromagnetic layer thickness of $10 \mathrm{~nm}$ in the case of top pinning and 6 $\mathrm{nm}$ in the case of bottom pinning. In each panel, data are included for both top and bottom pinned structures. 
$\mathrm{SiO}_{2}$ / Buffer / CoFe 5 / Cu 2.8 / CoFe 2.5 / IrMn 10 / Cap

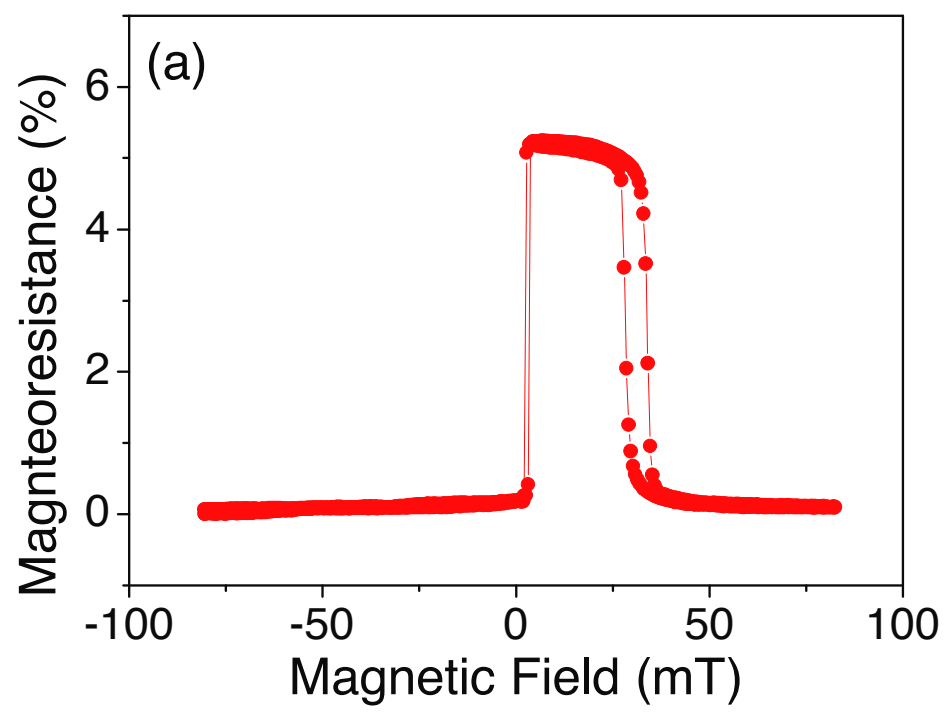

$\mathrm{SiO}_{2}$ / Buffer / IrMn 6 / CoFe 3.5 / Cu 2.8 / CoFe 5 / Cap

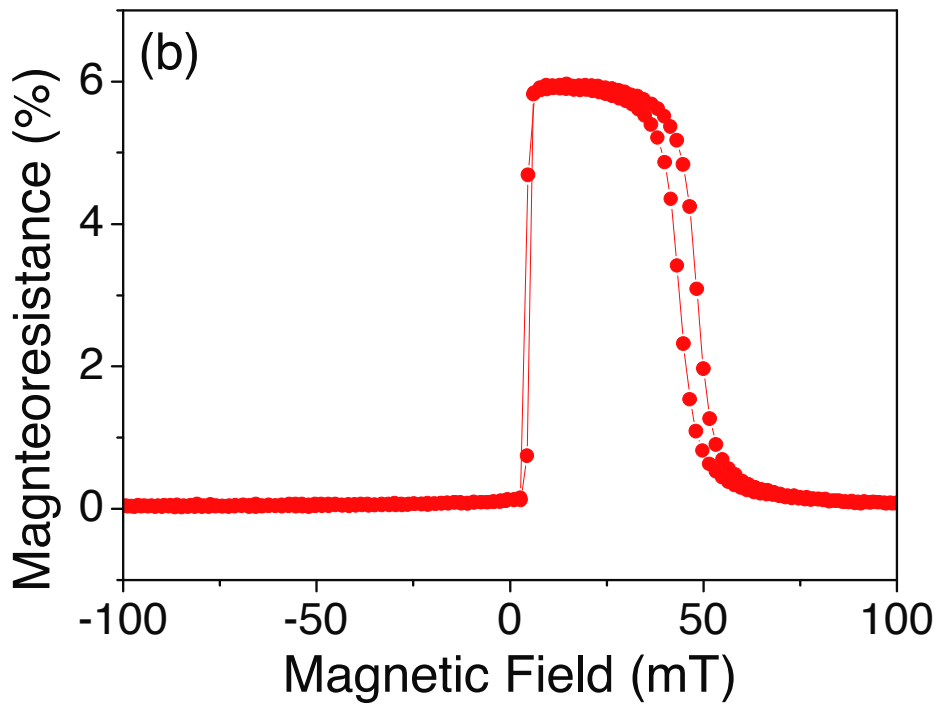

Figure 3. Examples of magnetoresistance curves for optimized a) top-pinned and b) bottom pinned single spin valves, described in the text. 


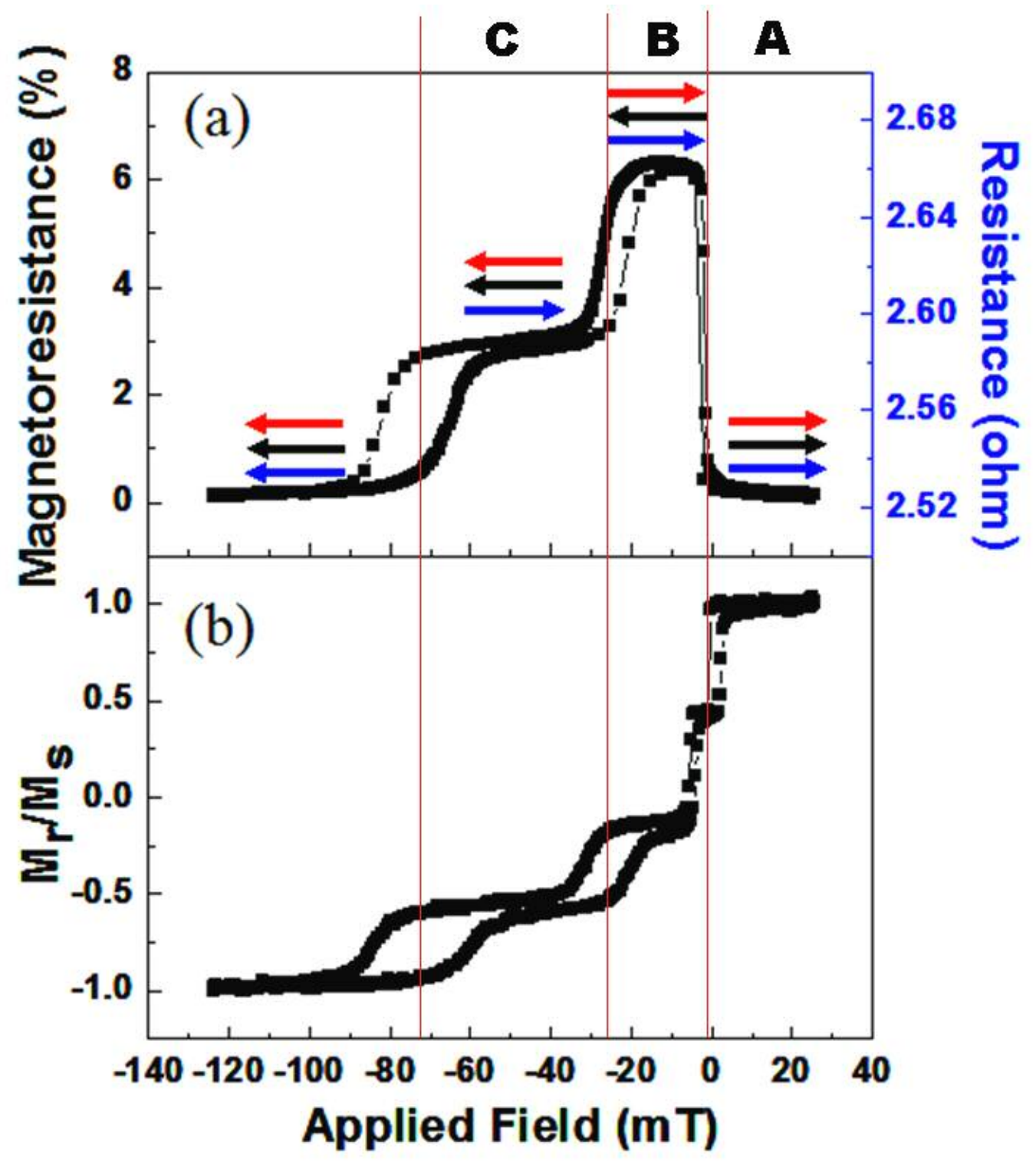

Figure 4. (a) The MR curve with current-in-plane (CIP) geometry and (b) M-H curve. Both curves show the stability of the intermediate state in field (sectioned as C). The extra switch near zero field in (b) but not in (a) is due to the presence of CoFe $3 \mathrm{~nm}$ in the buffer layer which does not affect the GMR signal. 


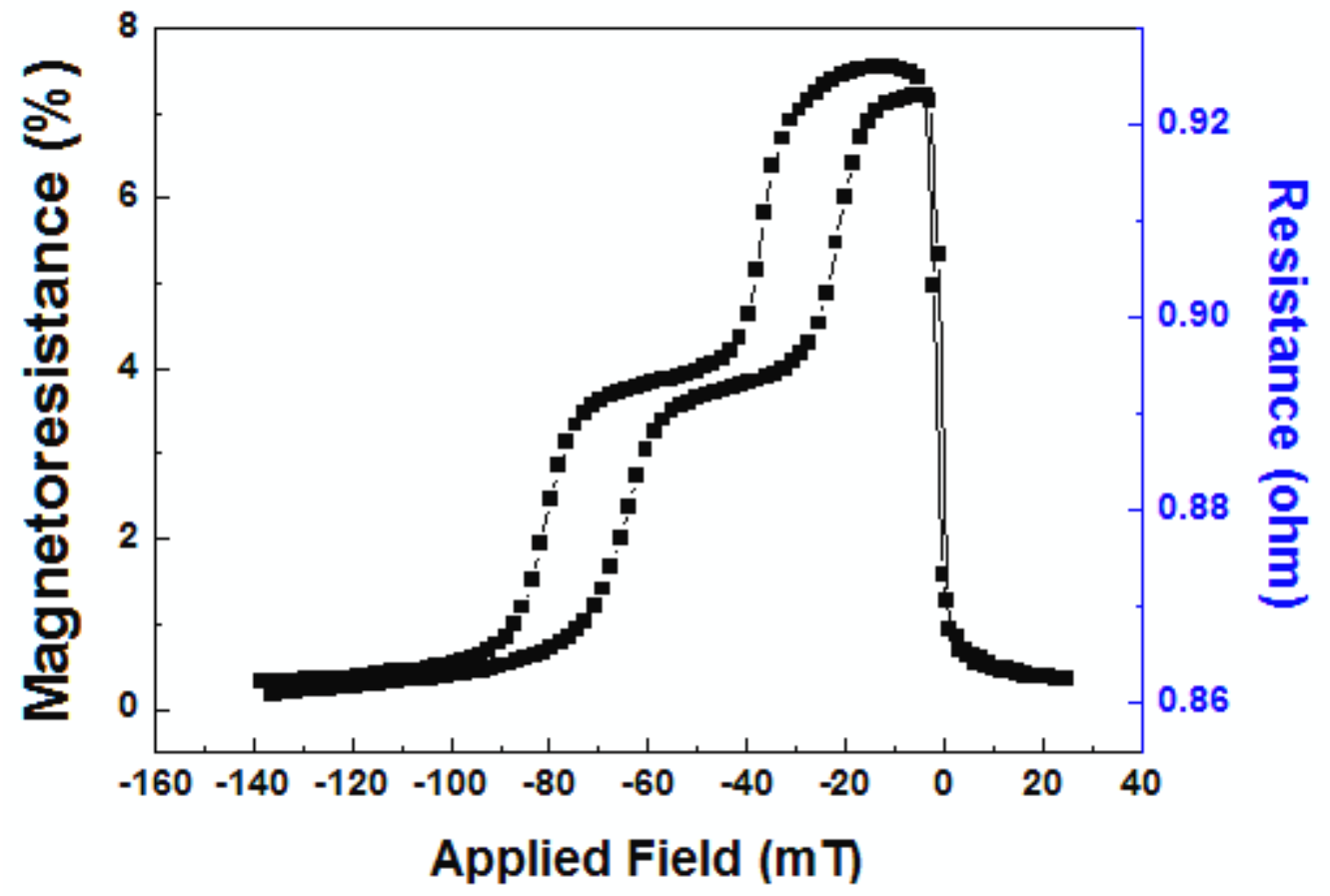

Figures 5. Resistance value and MR curve for the dual spin valve measured in CPP GMR. The sample was patterned to a $250 \mathrm{~nm}$ x $400 \mathrm{~nm}$ nanopillar as described in the text. 